\title{
The cultivation of epilepsy neurosurgeons: requirements and challenges
}

\author{
Kai Zhang ${ }^{1,3,45^{*}}$, Jianguo Zhang ${ }^{1,3,4,5}$, Yanshan $\mathrm{Ma}^{5}$, Xiaoqiu Shao ${ }^{2,5}$, Wenhan $\mathrm{Hu}^{3,4,5}$ and Baotian Zhao ${ }^{1}$
}

\begin{abstract}
Epilepsy surgery has been accepted as an effective treatment in drug-resistant focal epilepsy. However, the number of epilepsy neurosurgeons is not enough in China compared with the huge demand. The content of epilepsy neurosurgeon cultivation includes surgery itself as well as presurgical evaluation. In this article, we review not only different categories of epilepsy surgeries but also the presurgical evaluation approaches including non-invasive methods, such as semiology analysis, high-resolution structural and functional imaging techniques, scalp electroencephalography monitoring as well as invasive methods, such as electrocorticography (ECoG) and stereotactic electroencephalography (SEEG). We also discuss the importance of learning each method briefly. In addition, we suggest that epilepsy neurosurgeons should have a solid structural and functional anatomy basis as it plays pivotal role in localizing epileptogenic zone. Epilepsy neurosurgeons should also be familiar with the staged presurgical evaluation starting from the most preliminary examinations and progressing to more complex and invasive methods. During the diagnosis and therapeutic procedures, collaboration between epilepsy neurosurgeons and other specialists is essential. At last, the necessity of epilepsy neurosurgeon cultivation has been emphasized.
\end{abstract}

Keywords: Epilepsy neurosurgeon cultivation, Epilepsy surgery, Presurgical evaluation learning

\section{Background}

Epilepsy is "an enduring predisposition to generate epileptic seizures" [1]. The prevalence of epilepsy has been estimated at 0.4 to $0.8 \%$ globally [2]. Despite several kinds of anti-epileptic drugs (AEDs) have been developed currently, approximately one-third of all patients develop into chronic intractable epilepsy [3]. The etiology of epilepsy is an important prognostic factor. For example, the seizures of patients with stroke-related epilepsy are much more easily controlled by AEDs (54 \%) than seizures in patients with cortical dysplasia (24\%) or mesial temporal sclerosis (11 \%) [4]. Consideration of surgical therapy in appropriate intractable epilepsy patients is necessary because only a minority of these patients achieve seizure control by trying different AEDs.

After decades of practicing, surgery now is a wellaccepted and efficacious way to treat selected patients with drug-resistant epilepsy. In China, the number of epilepsy surgery performed each year is relatively few

\footnotetext{
* Correspondence: zhangkai62035@163.com

${ }^{1}$ Department of Neurosurgery, Beijing Tian Tan Hospital, Capital Medical University, No. 6, Tian Tan Xi Li, Beijing 100050, China

${ }^{3}$ Beijing Key Laboratory of Neurostimulation, Beijing 100050, China

Full list of author information is available at the end of the article
}

and could not meet the huge demand. Many intractable epilepsy patients cannot accept formal presurgical evaluation and lose the opportunity for surgical treatment. Qualified epilepsy centers cluster in a few metropolises, which causes inconvenience to patients from remote areas. Many neurosurgeons have inadequate knowledge about the presurgical evaluation process and the epilepsy surgeries. Under these circumstances, more qualified epilepsy neurosurgeons need to be cultivated to serve the patients better. The cultivation of a neurosurgeon is a time consuming and highly specialized process which demands dedication, understanding, and professionalism. Meanwhile, the cultivation of neurosurgeons specializing in epilepsy surgery has its own characteristics. There are several prerequisites for an epilepsy neurosurgeon, which will be discussed in the following context.

\section{The knowledge of anti-epileptic drugs (AEDs) management}

As the first line therapy of epilepsy, AEDs such as valproic acid, carbamazepine, valproic acid, phenytoin and lamotrigine play pivotal role in reducing seizures and alleviating symptoms. For patients who accept presurgical evaluation 
or surgery, drug therapy always needs adjustment during the process. Thus, epilepsy neurosurgeons should be familiar with the different properties of each AEDs, such as their interaction with other drugs [5], pharmacokinetic characteristics, and the indications for adding or withdraw of drugs.

Special population like children and pregnant women require unique medication. The application of AEDs in children can be more complicated compared with adults because it is challenging to evaluate certain side effects in children, especially the side effects that may affect cognitive functions. Besides, children are vulnerable to specific aspects of drug toxicity, for example, it has been reported that valproic acid can induce liver damage and barbiturates can cause behavioral disorders in children [6]. Seizures and AEDs can be harmful to the developing fetus [7]. Thus pregnant epilepsy patients need special care. Epilepsy neurosurgeons should keep in mind the AEDs management and their side effects.

\section{Necessity of mastering presurgical evaluation methods}

Accurate localization of the epileptogenic zone (EZ) before surgery is critical for a satisfying outcome. Seizure semiology can provide elementary information for clinical diagnosis and valuable topical diagnosis information [8]. Several auxiliary examinations could be applied during the presurgical evaluation process accordingly. There are non-invasive methods, including electroencephalograph (EEG), magnetic resonance imaging (MRI), magnetoencephalography (MEG), and positron emission tomographycomputed tomography (PET-CT). The invasive methods include electrocorticography (ECoG) and stereotactic electroencephalography (SEEG) [9]. Epilepsy neurosurgeons should master different approaches to evaluate the EZ as well as the underlying mechanisms respectively.

\section{Value of semiology in localization}

Epilepsy neurosurgeons should learn to analyze semiology in detail with the help of video-EEG before surgery. Common semiologic features include auras (e.g., somatosensory auras, visual auras, auditory auras, olfactory auras gustatory auras, autonomic auras, abdominal auras) [10], motor signs (positive and negative motor signs), tonic posturing (e.g., "fencing posture", "figure of 4"), automatisms (e.g., unilateral limb automatism, bipedal automatisms, ictal spitting, gelastic seizure, postictal cough, postictal nose wiping). The extant literature shows that some semiologic features are useful in lateralization but relatively fewer features help in localizing specific lobe. When the scalp EEG fails to detect seizure onset zone, the value of seizure semiology is better appreciated. Seizure semiology also has limitations in localizing seizure onset. Briefly, Seizure semiology is sometimes subjective and cannot determine the seizure onset zone $100 \%$ accurately.
Seizure semiology in some patients may signify the site of seizure propagation rather than origination. Besides, seizure semiology in patients with multifocal epilepsy can be complicated and variable, which compromises its localizing value. Due to the expression limitations, many semiologic features of seizures in adults are often absent in children. Under these circumstances, epilepsy neurosurgeons should assess the clinical semiology of recorded seizures together with clinical history, EEG, neuroimaging and neuropsychological information [8].

\section{Importance of learning electroencephalography}

Electroencephalography, including scalp EEG and invasive methods, such as ECoG or SEEG, provide the most specific approach to localize epileptogenic zone and has the best temporal resolution. The skill to implant subdural or depth electrode is essential for modern qualified epilepsy neurosurgeons because they need to conduct the operation themselves. Taking the depth electrode for example, the major procedures of implanting depth electrode from our experience include: (1) the surgeon drills the scalp and soft tissues and create a dent on the surface of the skull using the hand drill with $2.5-\mathrm{mm}$ triangular pyramid point drill bit; (2) then the surgeon fit the $2.5-\mathrm{mm}$ drill bit (fixed and guided by the stereotactic frame arch) in the dent in case it slides and then the skull is drilled open; (3) the monopolar coagulator probe is inserted to open the dura; (4) the implantation bolt is screwed into the small burr hole; (5) we calculate and measure the final depth distance for the electrode; (6) the trajectory is created by a stylet probe and then we insert the depth electrode as planned and fix it into the bolt, preventing electrode displacements and CSF leak. After the implantation, patients will accept longterm video-EEG monitoring in the ward.

The ability, which comes from training and much practicing, to interpret EEG results is highly demanding. During the cultivation period, epilepsy neurosurgeons could learn to interpret EEG results by participating the work of neuro-electrophysiology department and seek help from electrophysiologist and physician. The ictal video-EEG recording is quite important in localizing the EZ because it can record the ictal video and EEG simultaneously so that doctors can identify the type of seizures, and to pinpoint the EZ. Consistent interictal epileptiform discharges can also provide valuable information [11]. Sometimes, however, the semiology and EEG can be complex and even paradoxical due to the electrical spreading among different cortex areas, for example, an epileptic seizure arises from a "silent" cortex would remain asymptomatic until it influences the "eloquent" cortex, such as primary sensory areas or primary motor areas. To better define the EZ and detect the propagation of seizures in selected cases, invasive EEG 
techniques have been developed. SEEG has gained popularity these years because it facilitates precise recordings from deep cortical and subcortical structures, multiple noncontiguous lobes, and bilateral explorations while avoiding the need for large craniotomies [12]. The working basis of SEEG is anatomo-electro-functional hypothesis that is typically generated during the presurgical consultation conference based on the results of the former noninvasive tests [13]. Although scalp EEG recordings are less sensitive and have more artifacts than invasive methods, they still provide the best overview and the most efficient way for detecting the localization of the epileptogenic zone approximately. Cortical stimulation mapping can be performed under intraoperative waken-up anesthesia or extraoperatively through intracranial electrodes and it is helpful in determining the boarder of resection when the EZ is adjacent to or overlapping with functional cortex [14].

To sum up, epilepsy neurosurgeons should master the skill to implant subdural grid electrode or depth electrode themselves and meanwhile be familiar with the interpretation of results.

\section{Taking advantage of the advanced neuroimaging}

Neuroimaging, including structural imaging and functional imaging, frequently provides essential information for localizing EZ. The advances of neuroimaging have made it possible to reveal epileptogenic abnormalities that could not be identified previously. Structural imaging with high-resolution MRI of epilepsy patients has its own character that epilepsy neurosurgeons should notice. Common epileptogenic lesions such as hippocampal sclerosis, cavernous angioma, dysembryoplastic neuroepithelial tumor, and heterotopic gray matter can be identified by MRI [15]. However, attention should be paid that not all lesions seen on MRI images are epileptogenic, and certain EZ lies beyond the lesion [16]. Still, some radiographic lesions may be unrelated to the clinical seizures. For this reason, we cannot judge the EZ by neuroimaging alone. Other methods should also be used to verify that the radiographic lesion is indeed responsible for the clinical seizures. Sometimes changes on MRI images for epilepsy can be subtle or even negative, making presurgical evaluation difficult. For example, a pathology named focal cortical dysplasia (FCD) type I is frequently negative on MRI images [17]. Therefore, thin layer MRI scan and careful inspection combined with other clinical data are necessary.

In recent years, image post-processing techniques have been developed such as voxel-based morphology that facilitates detection of FCD and other anomalies. These techniques are helpful for the noninvasive identification of a structurally subtle lesion that can be surgically targeted [18]. Functional imaging such as PET-CT and ictal single-photon emission computed tomography (SPECT) are especially valuable when the MRI images are negative. Interictally, epileptogenic zones are frequently associated with reduced regional cerebral metabolism which can be detected by PET. With the help of MRI-PET coregistration, the potential EZ can be indicated and further invasive EEG studies can be guided [19]. Subtraction ictal single-photon emission computed tomography coregistered to MRI (SISCOM) has dramatically improved the efficacy of ictal SPECT. Blood flow may increase relatively during ictal state, and SISCOM technique can detect this discrepancy and display it on high-resolution MRI, indicating the potential EZ [20]. Epilepsy neurosurgeons should master the skill to analyze different kinds of images and learn their value and limitations. Thus, epilepsy neurosurgeons should spend some time in the imaging department to learn these skills.

\section{Neuropsychological assessments}

Neuropsychological assessments are essential for diagnosis and epilepsy-related cognitive comorbidities and treatment evaluation. A neuropsychological assessment should sample many different cognitive functions, such as intelligence quotient (IQ), attention, visual perception, auditory perception, spatial ability, language, fluency, memory, planning, concept formation, social behavior and personality, somatosensory and motor functions. For those who would accept epilepsy surgery, neuropsychological assessments provide accessory information regarding the localization and lateralization of epilepsy-associated cognitive impairment [21]. For example, left temporal lobe epilepsy is strongly related with impairment of verbal episodic memory. Frontal lobe epilepsy patients performed below normal on fluency, executive functions, global and verbal intelligence [22]. Although postoperative seizure free is the major task, we also aim at preservation or even improvement of cognitive functions. Neuropsychological tests need to be conducted before and after operation. Through a set of accumulated cognitive results, epilepsy neurosurgeons could gradually acquire the ability to determine the safe boundary of resection of cortex to minimally diminish the risk of cognition decline. Postoperative cognition decline can be reduced when surgery is restricted to tissues which is not involved in specific function and has greater cerebral plasticity and greater capacities for compensation [23].

Neuropsychological assessment occupies a unique position among presurgical evaluation methods because it is the only method for understanding the cognition impact of epilepsy and epilepsy surgery. The tests results may support a number of important decisions in medical management, such as the identification of candidates for surgery, potential risk, benefits and prognosis. Epilepsy neurosurgeons should realize the importance of neuropsychological assessments and apply them selectively. 


\section{Solid structural and functional anatomy basis}

Anatomy knowledge is the working basis for any neurosurgeon. Since EZ can locate in any lobe of the cerebral cortex and manifest various semiology accordingly, epilepsy neurosurgeons must master the structural anatomy as well as functional neuroanatomy. It has been widely accepted that symptomatogenic zone located in different lobe can generate various semiology that are highly related to the corresponding function cortex. For example, the contralateral primary sensory cortex is usually the symptomatogenic zone of somatosensory auras of unilateral extremities and the symptomatogenic zone for the visual hallucinations are Broadmann's area 17 and 18 [10]. Even in the same cerebral lobe, semiology can be divided into subgroups according to different clinical features and correlate with different cortical areas within a cerebral lobe through cluster analysis [24]. The complexity of EZ localization requires epilepsy neurosurgeons to have an integral view of structural neuroanatomy and detailed functional neuroanatomy.

Brodmann areas have been widely used in regions of the cerebral cortex, defined by its cytoarchitecture, or histological structure and organization of cells since the first introduction in 1909 by German anatomist Korbinian Brodmann. It has also been widely used in EZ localization because Brodmann areas are relatively more specific and constant than certain anatomy structures. More importantly, these areas are frequently related with specific brain function which provides valuable information during semiology analysis.

Our brain is a functional network, and cortex areas are inter-connected by white matter fasciculi. The main fasciculi in our brain include (1) the superior longitudinal (arcuate) fasciculus, (2) the inferior longitudinal fasciculus, (3) the superior fronto-occipital (subcallosal) fasciculus, (4) the inferior fronto-occipital fasciculus, (5) the uncinate fasciculus, (6) the cingulum, (7) the anterior commissure, (8) the corpus callosum, (9) the internal capsule, and (10) the fornix [25]. Although the role of these fasciculi in the spreading of ictal epileptic discharges needs further investigation, the structure and physiological function should be well known by epilepsy neurosurgeons.

\section{Different categories of epilepsy surgery}

Epilepsy surgeries can generally be divided into resective surgery, aiming at total removal of the epileptogenic area without causing permanent neurological deficits, and palliative surgery, such as vagal nerve stimulation (VNS), corpus callosotomy, and multiple subpial transections [3]. As for the resective surgeries, epilepsy neurosurgeon should remember that it is essential to preserve certain vessels overlying the cortex to be removed and the subcortical white matter underlying the resected cortex in some circumstance [26]. During the operation, to define the EZ after craniotomy can be challenging sometimes and so is the subsequent tailored resection. With the help of neuronavigation, neurosurgeons get much easier access to the planned resective area. Surface morphology of the brain should be carefully identified to help reach the planned EZ.

VNS is a prevailing palliative surgery which is an important option for those unsuitable for resective surgery after formal presurgical evaluation. Since the U.S. Food and Drug Administration approved this device for refractory epilepsy in adult and adolescents in July 1997, this modality is the most widely used nonpharmacological treatment for drug-resistant epilepsy now. However, the underlying mechanisms of VNS remain poorly understood [27]. Other neuromodulation methods, such as chronic anterior thalamic stimulation [28], and responsive neurostimulator [29] are under clinical trials and have shown promising efficacy preliminarily. During the cultivation period, epilepsy neurosurgeons should be familiar with different kinds of epilepsy surgery and their indications as well as their contraindications. At the same time, attentions should be paid on the progress of epilepsy surgery.

\section{Cooperation between epilepsy neurosurgeon and other specialists}

During the cultivation period and later, epilepsy neurosurgeons should never work alone. Due to the specialty limitations, epilepsy neurosurgeons, physicians, pediatricians should work as a team for diagnosis and planning for further therapeutic alternatives. In this team, epilepsy neurosurgeon is responsible for suggesting the surgical candidate to accept formal presurgical evaluation and early epilepsy surgery if necessary. Early epilepsy surgery is proposed because early intervention in the course of epilepsy may avoid the devastating psychosocial effects of chronic epilepsy and improve the long-term quality of life [30]. Besides, early surgical intervention in children with focal epilepsies may also prevent the psychosocial morbidity and neuropsychological decline that are caused by drug-resistant epilepsy [31].

The presurgical evaluation is a staged process and needs the participation of neurosurgeons, physicians, pediatricians and neuroelectrophysiology doctors. The first stage always includes detailed clinical history gathering and physical examination, video-EEG monitoring, highresolution MRI images, neuropsychological testing, and assessment of psychosocial functioning. The second stage may include functional studies, such as PET and SISCOM. The last stage, if necessary, mainly referred to intracranial monitoring such as ECoG or SEEG [32]. Clinical data obtained from each stage should be analyzed carefully and guide the subsequent evaluation. The proper selection of various investigations and their accurate interpretation at 
each stage is essential to ensure a successful outcome. Epilepsy neurosurgeons should take part in these stages and express their opinions about the surgery affairs during the cultivation period.

\section{The importance of self-improvement for epilepsy neurosurgeons}

Epilepsy neurosurgeons should realize that although progress in technologies, such as electroencephalography and neuroimaging, has improved our ability to accurately localize the EZ, however, failure of epilepsy surgery still cannot be fully avoided. Various hypotheses have been put forward like wrong localization of the epileptogenic zone, insufficient resection, dual pathology, very widespread epileptogenic zones [33]. The accurate prediction of individual outcomes after epilepsy surgery still challenges epilepsy neurosurgeons [34]. Long-term seizure free rate of resective surgery ranges from 27 to $66 \%$ according to different type of surgery [35]. Epilepsy neurosurgeons should take the duty of improving surgical outcome and avoiding complications at the same time. They should also conduct some practical clinical trial as well as basic research during the cultivation period. The ability to search and learn from literature are critical in improving professional level. Attending meetings and communicating with other epilepsy neurosurgeons are also helpful.

\section{Conclusions}

Surgical therapy has the potential to eliminate seizure recurrence and improve the quality of life in selected patients with drug-resistant focal epilepsy. As the key role in epilepsy surgery, epilepsy neurosurgeons should not only be capable of performing successful surgeries but also need to be familiar with different approaches to evaluate the EZ as well as the underlying mechanisms. The process of cultivating a qualified epilepsy neurosurgeon is time-consuming and challenging. In China, qualified epilepsy neurosurgeons are far from enough and many drug-resistant focal epilepsy patients could not accept formal presurgical evaluation and lose the opportunity for surgery. Under such circumstances, more epilepsy neurosurgeon should be cultivated to serve the epilepsy patients better.

\section{Abbreviations \\ AEDs, anti-epileptic drugs; ECoG, electrocorticography; $E E G$, electroencephalograph; EZ, epileptogenic zone; FCD, focal cortical dysplasia; MEG, magnetoencephalography; MRI, magnetic resonance imaging; PET-CT, positron emission tomography-computedtomography; SEEG, stereotactic electroencephalography; SISCOM, subtraction ictal single- photon emission computed tomography coregistered to MRI; SPECT, single- photon emission computed tomography; VNS, vagal nerve stimulation.}

\section{Acknowledgements}

We gratefully acknowledge the hard work of the team in our epilepsy center located in Peking University First Hospital Fengtai Hospital. Without their help, it would be hard to finish this work.

\section{Funding}

This work was supported by grants from the National Natural Science Foundation of China (No. 81171218), Beijing Municipal Administration of Hospitals Clinical Medicine Development of Special Funding (code: XMLX201304; code: ZYLX201305).

\section{Availability of data and materials}

This paper is a review article. Referred literature in this paper has been listed in the references part. The datasets supporting the conclusions of this article is are available online by searching the PubMed. Some original points in this article come from the clinical practice in our epilepsy center and the authors' experiences.

\section{Authors' contributions}

$K Z$, JGZ conceived the topic and plot of this paper, analyzed the materials and wrote the paper. $\mathrm{WHH}$ and BTZ searched the relevant literatures and enriched the topic. YSM and XQS put forward critical suggestions based on their perspective about this paper and revise it as well. All authors have contributed to, seen and approved the manuscript.

\section{Authors' information}

Zhang Kai, M.D., postgraduate supervisor, vice director of department of stereotactic and functional neurosurgery, Beijing Tian Tan Hospital, Capital Medical University. Specializing in presurgical evaluation and epilepsy surgery.

\section{Competing interests}

The authors declare that they have no competing interests.

\section{Consent for publication}

Not applicable.

\section{Ethics approval and consent to participate} Not applicable.

\section{Author details}

${ }^{1}$ Department of Neurosurgery, Beijing Tian Tan Hospital, Capital Medical University, No. 6, Tian Tan Xi Li, Beijing 100050, China. ²Department of Neurology, Beijing Tian Tan Hospital, Capital Medical University, Beijing 100050, China. ${ }^{3}$ Beijing Key Laboratory of Neurostimulation, Beijing 100050 , China. ${ }^{4}$ Stereotactic and Functional Neurosurgery Laboratory, Beijing Neurosurgical Institute, Capital Medical University, Beijing 100050, China. ${ }^{5}$ Epilepsy Center, medical alliance of Beijing Tian Tan Hospital, Peking University First Hospital Fengtai Hospital, Beijing 100000, China.

Received: 13 December 2015 Accepted: 25 May 2016

Published online: 18 June 2016

\section{References}

1. Duncan JS, et al. Adult epilepsy. Lancet. 2006;367:1087-100.

2. Hauser WA, et al. Seizure recurrence after a 1st unprovoked seizure: an extended follow-up. Neurology. 1990;40:1163-70.

3. Schuele SU, Lüders HO. Intractable epilepsy: management and therapeutic alternatives. Lancet Neurol. 2008;7:514-24.

4. Semah F, Picot MC, Adam C, et al. Is the underlying cause of epilepsy a major prognostic factor for recurrence? Neurology. 1998;51:1256-62.

5. Rivers F, O'Brien TJ, Callaghan R. Exploring the possible interaction between anti-epileptic drugs and multidrug efflux pumps; in vitro observations. Eur J Pharmacol. 2008:598:1-8

6. Perucca E. Pharmacological problems in the management of epilepsy in children. Seizure. 1995;4:139-43.

7. Yerby MS, Kaplan P, Tran T. Risks and management of pregnancy in women with epilepsy. Cleve Clin J Med. 2004;71 Suppl 2:S25-37.

8. So EL. Value and limitations of seizure semiology in localizing seizure onset. J Clin Neurophysiol. 2006;23:353-7.

9. Rosenow F, Luders H. Presurgical evaluation of epilepsy. Brain. 2001;124:1683-700

10. Tufenkjian K, Luders HO. Seizure semiology: its value and limitations in localizing the epileptogenic zone. J Clin Neurol. 2012;8:243-50.

11. Engel Jr J. A practical guide for routine EEG studies in epilepsy. J Clin Neurophysiol. 1984;1:109-42. 
12. Cossu M, et al. Stereoelectroencephalography in the presurgical evaluation of focal epilepsy: A retrospective analysis of 215 procedures. Neurosurgery. 2005;57:706-18

13. Gonzalez-Martinez J, et al. Stereoelectroencephalography in the "difficult to localize" refractory focal epilepsy: early experience from a North American epilepsy center. Epilepsia. 2013;54:323-30

14. Luders $\mathrm{H}$, et al. Localization of cortical function: new information from extraoperative monitoring of patients with epilepsy. Epilepsia. 1988;29 Suppl 2:S56-65.

15. Wehner $\mathrm{T}$, Luders $\mathrm{H}$. Role of neuroimaging in the presurgical evaluation of epilepsy. J Clin Neurol. 2008:4:1-16.

16. Sevy A, et al. Beyond the lesion: the epileptogenic networks around cavernous angiomas. Epilepsy Res. 2014;108:701-8.

17. Blumcke I, et al. The clinicopathologic spectrum of focal cortical dysplasias: a consensus classification proposed by an ad hoc task force of the ILAE diagnostic methods commission. Epilepsia. 2011;52:158-74.

18. Wang ZI, et al. Linking MRI postprocessing with magnetic source imaging in MRI-negative epilepsy. Ann Neurol. 2014;75:759-70

19. Fernandez S, et al. PET/MRI and PET/MRI/SISCOM coregistration in the presurgical evaluation of refractory focal epilepsy. Epilepsy Res. 2015;111:1-9.

20. Knowlton RC. The role of FDG-PET, ictal SPECT, and MEG in the epilepsy surgery evaluation. Epilepsy Behav. 2006;8:91-101.

21. Jones-Gotman M, et al. The contribution of neuropsychology to diagnostic assessment in epilepsy. Epilepsy Behav. 2010;18:3-12.

22. Ljunggren $\mathrm{S}$, et al. Cognitive outcome two years after frontal lobe resection for epilepsy-a prospective longitudinal study. Seizure. 2015:30:50-6.

23. Helmstaedter C. Neuropsychological aspects of epilepsy surgery. Epilepsy Behav. 2004;5 Suppl 1:S45-55.

24. Bonini F, et al. Frontal lobe seizures: from clinical semiology to localization. Epilepsia. 2014;55:264-77.

25. Catani $\mathrm{M}$, et al. Virtual in vivo interactive dissection of white matter fasciculi in the human brain. Neuroimage. 2002;17:77-94.

26. Girvin, JP. Corticectomy. In: Girvin, J.P., editor. Operative techniques in epilepsy. Switzerland: Springer International Publishing; 2015. p. 107-23.

27. Lulic D, et al. Vagus nerve stimulation. Neurosurg Focus. 2009;27(3):E5.

28. Kerrigan JF, et al. Electrical stimulation of the anterior nucleus of the thalamus for the treatment of intractable epilepsy. Epilepsia. 2004;45:346-54.

29. Fountas KN, et al. Implantation of a closed-loop stimulation in the management of medically refractory focal epilepsy: a technical note. Stereotact Funct Neurosurg. 2005;83:153-8.

30. Kwan P, Brodie MJ. Early identification of refractory epilepsy. N Engl J Med. 2000:342:314-9.

31. Loddenkemper T, et al. Developmental outcome after epilepsy surgery in infancy. Pediatrics. 2007;119:930-5.

32. Rathore C, Radhakrishnan K. Concept of epilepsy surgery and presurgical evaluation. Epileptic Disord. 2015;17(1):19-31.

33. Harroud A, et al. Temporal lobe epilepsy surgery failures: a review. Epilepsy Res Treat. 2012;2012:201651.

34. Wrench JM, et al. Current challenges in the practice of epilepsy surgery. Epilepsy Behav. 2011;22:23-31.

35. Tellez-Zenteno JF, Dhar R, Wiebe S. Long-term seizure outcomes following epilepsy surgery: a systematic review and meta-analysis. Brain. 2005;128. 1188-98.

\section{Submit your next manuscript to BioMed Central and we will help you at every step:}

- We accept pre-submission inquiries

- Our selector tool helps you to find the most relevant journal

- We provide round the clock customer support

- Convenient online submission

- Thorough peer review

- Inclusion in PubMed and all major indexing services

- Maximum visibility for your research

Submit your manuscript at www.biomedcentral.com/submit

) Biomed Central 